\title{
Transtornos neuropsiquiátricos em idosos institucionalizados no sul de Santa Catarina (Brasil)
}

\section{Neuropsychiatric disorders in institutionalized elderly in south of Santa Catarina (Brazil)}

\author{
Alceu V. Panini'; Jaqueline F. Menegasso'; Laryce Galvan'; Viviane P. Feldens²; Daniele B. Vinholes ${ }^{3}$
}

\begin{abstract}
RESUMO
Contexto: $O$ processo de envelhecimento está associado às doenças que podem comprometer o bom funcionamento do sistema nervoso central. Objetivo: Avaliar característica de declínio cognitivo e prevalência de sintomas depressivos e sua associação com dados sociodemográficos de idosos institucionalizados em três instituições de longa permanência do sul de Santa Catarina. Métodos: Estudo transversal, avaliando todos idosos institucionalizados em Tubarão e Laguna/SC. Participaram da presente pesquisa 66 idosos, com idade maior ou igual a sessenta anos, de ambos os gêneros. Para coleta de dados utilizou-se um questionário padronizado abordando dados sociodemográficos, a Escala de Depressão Geriátrica reduzida (EDG-15) e o Mini-Exame do Estado Mental. Resultados: A população estudada era predominantemente feminina, viúva e média de idade $77,3 \pm 9,9$ anos. Sintomas depressivos estiveram presentes em $62,1 \%$ dos idosos. Possível déficit cognitivo esteve presente em $37,87 \%$ da população. Conclusão: Alta prevalência de sintomas depressivos e déficit cognitivo estiveram presentes nos idosos residentes nas instituições de longa permanência pesquisadas. Diante de tais fatos, o serviço de saúde do Brasil deve estar preparado para avaliar e tratar tais pacientes, a fim de melhorar a qualidade de vida dos idosos institucionalizados.
\end{abstract}

Palavras-chave: Idosos. Transtornos Neuropsiquiátricos. Institucionalização. Depressão. Déficit Cognitivo.

\section{Introdução}

O envelhecimento pode ser entendido como um processo dinâmico e progressivo, onde ocorrem alterações da morfologia humana, alterações funcionais, bioquímicas e psicológicas que determinam a progressiva perda da capacidade de adaptação do indivíduo ao meio ambiente, levando a maior vulnerabilidade $\mathrm{e}$ maior incidência de processos patológicos. ${ }^{1}$

A população de indivíduos idosos está aumentando mundialmente, inclusive nos países em desenvolvimento. ${ }^{2}$ A classificação das faixas etárias segundo a Organização Mundial de Saúde (OMS) é de acordo com o grau de desenvolvimento de cada país. Em
1. Graduação em Medicina, Universidade do Sul de Santa Catarina. Membro da Liga Acadêmica de Psiquiatria da UNISUL. Tubarão, SC, Brasil.

2. Doutorado em Psicologia Médica, Professora do curso de graduação em Medicina da Universidade do Sul de Santa Catarina, Tubarão, SC, Brasil

3. Doutorado em Epidemiologia - UFRGS. Professora do curso de graduação em Medicina da Universidade do Sul de Santa Catarina, Tubarão, SC, Brasil.
Correspondência Daniele Botelho Vinholes Rua Guararapes 70 apt $^{\circ} 701$ CEP 90690-340 Porto Alegre/RS Email: dvinholes@terra.com.br

Artigo recebido em 27/03/2013 Aprovado para publicação em 03/10/2013 
países em desenvolvimento, como o Brasil, são considerados idosos, indivíduos com idade igual ou superior a sessenta anos. ${ }^{3}$

Os idosos representam uma parcela da população mais susceptível a uma série de doenças e distúrbios, entre eles os distúrbios neuropsiquiátricos. As síndromes depressivas, demenciais e o declínio cognitivo representam os transtornos neuropsiquiátricos mais prevalentes em indivíduos idosos. ${ }^{4}$

O processo de envelhecimento está associado às doenças que podem comprometer o bom funcionamento do sistema nervoso central. Dentre elas, as enfermidades neuropsiquiátricas, tais como a depressão e déficit cognitivo. ${ }^{5}$

O declínio cognitivo nos pacientes idosos é caracterizado por lentidão leve, generalizada e perda da precisão, quando tais idosos são comparados com pessoas mais jovens. ${ }^{6}$ Ainda, no que tange habilidade cognitiva, pacientes idosos com um QI elevado, apresentam uma proteção para demência. ${ }^{4}$ A cognição é uma definição da atividade mental, englobando habilidades como sentir, pensar, perceber, lembrar, raciocinar, formar estruturas complexas de pensamento e a capacidade de produzir respostas às solicitações e estímulos externos. $^{7}$

A OMS considerou depressão como a quarta causa específica de incapacidade social comparativamente com outras doenças durante a década de 90 . A previsão para 2020 é que se torne a segunda causa de incapacidade em países desenvolvidos, e a primeira nos países em desenvolvimento, como no Brasil. ${ }^{8}$

Um dos métodos mais utilizados para identificação de sintomas depressivos na velhice é a Escala de Depressão Geriátrica de Yesavage (EDG). Essa escala mostrou-se como um indicador relativamente estável e pode ser utilizada para detecção de casos de depressão no idoso e monitoramento da gravidade dos seus sintomas. ${ }^{9}$

No que tange as habilidades cognitivas, o MiniExame do Estado Mental (MEEM) é um instrumento que auxilia na investigação e na monitoração da evolução de possíveis déficits cognitivos em pessoas com risco de demência. Desde que foi proposto por Folstein et al., ${ }^{10}$ vem sendo amplamente utilizado no mundo. No Brasil, o MEEM foi inicialmente proposto por Bertolucci et al., que observaram que o escore total do MEEM dependia do nível educacional. ${ }^{11}$

Muitas vezes, pela falta de condições da família, tanto financeiras como psicológicas, muitos pacientes idosos são institucionalizados em locais especiali- zados nos cuidados de pessoas com mais de sessenta anos de idade. ${ }^{12}$ Os asilos são a modalidade mais antiga e universal de atendimento ao idoso fora do convívio familiar. Esses locais de atendimento aos idosos apresentam fatores negativos, como: isolamento e inatividade física e mental. Esse isolamento social leva ao idoso à perda da identidade, de liberdade, de autoestima, solidão e, muitas vezes, à recusa da própria vida, o que pode justificar a alta prevalência de depressão. Portanto, a institucionalização asilar é uma situação estressante e desencadeadora de depressão. ${ }^{13}$

A depressão pode ser fator de risco para o desenvolvimento de declínio cognitivo, ${ }^{14}$ particularmente, entre idosos institucionalizados, e a institucionalização e a perda de atividade parecem afetar a depressão e a cognição. Tendo em vista que a depressão e o declínio cognitivo são causas importantes de morbidade, sofrimento, incapacidade e afetam sensivelmente a qualidade de vida dos indivíduos que sofrem desses transtornos, torna-se necessário o desenvolvimento de estudos que busquem conhecer a realidade da saúde mental dos idosos institucionalizados, com a finalidade de diagnosticar precocemente e tratar de maneira adequada tais transtornos neuropsiquiátricos.

Este estudo tem por objetivo avaliar sintomas de declínio cognitivo e prevalência de sintomas depressivos e suas relações com as características demográficas e socioeconômicas dos idosos institucionalizados.

\section{Material e métodos}

Trata-se de um estudo transversal em que foram avaliados todos os idosos institucionalizados nas duas Instituições de Longa Permanência (ILP) da cidade de Tubarão/SC e outra localizada na cidade de Laguna/SC. A escolha dessas três instituições teve por finalidade buscar uma amostra representativa, sendo a totalidade de ILPS localizadas nos municípios com mais de 50 mil habitantes da região do AMUREL (Associação dos Municípios da Região de Laguna). Foram incluídos homens e mulheres, com idade igual ou superior a 60 anos, que consentiram com a participação através de consentimento livre e esclarecido. Como critérios de exclusão, podemos citar incapacidade de entender o questionário ou alterações motoras, que prejudiquem o desempenho no estudo ou que impeçam de entender e responder aos questionamentos, além de pacientes já diagnosticados com déficit cognitivo previamente. 
A coleta de dados aconteceu no período de janeiro a abril de 2012. As entrevistas foram agendadas previamente com a direção das ILPs para não interferirem com o andamento das atividades normais das mesmas. Após esclarecimentos sobre o objetivo do estudo e aceitabilidade em participar da pesquisa, foi aplicado um questionário padronizado em forma de entrevista individual. Durante a entrevista, foi aplicado o Mini-Exame do Estado Mental (MEEM) desenvolvido por Folstein e McHugh ${ }^{10}$ em 1975 e traduzido por Bertolucci et al. $(1994)^{11}$ que é composto por diversas questões agrupadas em sete categorias, sendo que cada uma delas objetiva avaliar déficits das funções cognitivas específicas: orientação para o tempo (5 pontos), orientação para o local (5 pontos), registro de três palavras ( 3 pontos), atenção e cálculo ( 5 pontos), lembrança de três palavras (3 pontos), linguagem (8 pontos) e capacidade construtiva visual (1 ponto). $\mathrm{O}$ escore do MEEM pode variar de um mínimo de 0 até o total máximo de 30 pontos. O MEEM varia conforme o grau de instrução do idoso, sendo que o ponto de corte para idosos sem escolaridade é 13 pontos; 18 para escolaridade média (1-8 anos de estudos) e 26 para escolaridade alta (mais que 8 anos de estudos).

Após foi aplicada a Escala de Depressão Geriátrica em versão reduzida de Yesavage (EDG-15). ${ }^{9} \mathrm{~A}$ EDG-15 é amplamente utilizada e validada como instrumento diagnóstico de depressão em pacientes idosos. É um teste para detecção de sintomas depressivos no idoso, com 15 perguntas (Sim/Não) onde o resultado de 5 ou mais pontos sugere presença de sintomas depressivos, sendo que o escore igual ou maior que 11 caracterizam-se sintomas mais graves. ${ }^{15}$ Os dados referentes à medicação foram coletados diretamente dos prontuários dos idosos.

A pesquisa foi realizada após autorização das Instituições e após aprovação do Comitê de Ética em Pesquisa da Universidade do Sul de Santa Catarina. (Protocolo 11.243.4.01.III)

Os dados foram digitados no programa Epinfo 3.5.1. e avaliados no programa SPSS 18.0 (Chicago / USA), através de uma análise descritiva, primeiramente, utilizando números absolutos e frequiências para as variáveis categóricas e média e desvio-padrão para as variáveis contínuas. Para avaliar a associação entre as variáveis foram utilizados os seguintes testes de associação: teste do qui-quadrado para testar associação entre variáveis categóricas e teste t de Student e ANOVA para testar a associação quando uma das variáveis for contínua. O nível de significância utilizado foi de $95 \%$.

\section{Resultados}

A amostra inicial foi composta por 123 institucionalizados em três Instituições de Longa Permanência (ILP) no sul de Santa Catarina desses, 57 foram excluídos, pelos seguintes motivos: 18 idosos por apresentarem incapacidade de compreensão das perguntas, provavelmente causado por um déficit cognitivo severo. Ainda, haviam 17 pessoas institucionalizadas em ambiente especializado ao atendimento ao idoso, que não estavam na faixa etária maior ou igual aos sessenta anos, sendo esses não incluídos na pesquisa. Foram excluídos ainda: nove idosos que apresentaram alteração sensorial que prejudicasse o desempenho na pesquisa, quatro idosos hospitalizados durante o período da pesquisa, quatro idosos com alterações motoras significativas, quatro idosos recusaram a participar e um idoso faleceu durante o período da pesquisa.

Foram entrevistados 66 idosos, a maioria do gênero feminino (65,2\%), com idade entre 61-100 anos, sendo a média de idade de 77,3 9 9,9 anos. A maioria dos idosos era caucasianos, com religião católica e estado civil viúvo. Quanto ao nível de escolaridade, havia idosos que nunca frequentaram ambiente escolar e idosos com 15 anos de estudos, sendo a média em anos de estudos de 3,09 $\pm 2,61$.

Quanto ao uso de antidepressivos, a maioria dos idosos $(56,1 \%)$ informou que não fazia uso e 27,3\% informaram que faziam uso de medicação antidepressiva. Quanto ao histórico tabagista, $56,1 \%$ eram nãofumantes e a maioria dos idosos entrevistados não ingere e nunca ingeriram excessivamente bebidas de álcool. O tempo de permanência nas instituições va-

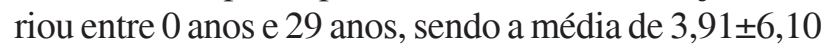
anos. O número de filhos variou de 0-11 filhos, sendo a média de 1,95 $\pm 2,36$ filhos. (Tabela 1 )

A pontuação na EDG variou entre $0-13$ pontos com média de $5,77 \pm 3,3$ pontos. Observou-se que três idosos $(4,5 \%)$ apresentavam sintomas depressivos severos, $11(16,7 \%)$ sintomas depressivos moderados, $27(40,9 \%)$ sintomas depressivos leve, totalizando $62,1 \%$ de idosos com alguma sintomatologia referente à depressão. 25 idosos $(37,9 \%)$ não apresentaram sintomas depressivos.

Nos idosos que fazem uso de medicação antidepressiva verificou-se que a pontuação do EDG foi maior em relação aos que não faziam uso de medicação antidepressiva $[7,6 \pm 3,2$ pontos $v s 4,7 \pm 2,8$ pontos, respectivamente (valor $\mathrm{p}=0,01)]$. 


\section{Tabela 1}

Características dos idosos residentes em três Instituições de Longa Permanência no sul de Santa Catarina.

\begin{tabular}{|c|c|c|}
\hline Características & Categorias & $N(\%)$ ou média \pm desvio-padrão \\
\hline \multirow[t]{2}{*}{ Gênero } & Feminino & $43(65,2)$ \\
\hline & Masculino & $23(34,8)$ \\
\hline Idade & \multicolumn{2}{|c|}{77,3 anos $\pm 9,9$ anos } \\
\hline \multirow[t]{2}{*}{ Raça } & Caucasianos & $57(86,4)$ \\
\hline & Não caucasiano & $9(13,6)$ \\
\hline \multirow[t]{3}{*}{ Religião } & Católica & $50(75,8)$ \\
\hline & Evangélica & $11(16,7)$ \\
\hline & Indeterminada & $5(7,5)$ \\
\hline \multirow[t]{4}{*}{ Estado Civil } & Viúvos & $26(39,4)$ \\
\hline & Solteiro & $22(33,3)$ \\
\hline & Divorciado & $15(22,7)$ \\
\hline & Casado & $3(4,5)$ \\
\hline Escolaridade & \multicolumn{2}{|c|}{$3,09 \pm 2,61$ anos } \\
\hline \multirow[t]{3}{*}{ Escolaridade } & Sem escolaridade & $8(12,1)$ \\
\hline & Média escolaridade & $56(84,8)$ \\
\hline & Alta escolaridade & $2(3,0)$ \\
\hline \multirow[t]{3}{*}{ Uso de Antidepressivos } & Sim & $18(27,3)$ \\
\hline & Não & $37(56,1)$ \\
\hline & Não Sabem & $11(16,7)$ \\
\hline \multirow[t]{3}{*}{ Tabagismo } & Não-Fumantes & $37(56,1)$ \\
\hline & Fumantes & $9(13,6)$ \\
\hline & Ex-fumantes & $20(30,3)$ \\
\hline \multirow[t]{2}{*}{ Uso de Álcool } & Sim & $17(25,8)$ \\
\hline & Não & $49(74,2)$ \\
\hline Tempo de ILP & \multicolumn{2}{|c|}{$3,91 \pm 6,1$ anos } \\
\hline Filhos & \multicolumn{2}{|c|}{$1,95 \pm 2,36$ filhos. } \\
\hline
\end{tabular}


O tempo de permanência na ILP não apresentou associação com a EDG (valor $\mathrm{p}=0,67$ ), assim como gênero (valor $\mathrm{p}=0,26$ )

O escore do MEEM variou entre 7-28 pontos, sendo a média $18,34 \pm 5,00$ pontos. As variáveis gênero, idade e tempo de permanência na ILP não apresentaram relação significativa com o escore do MEEM.

Analisando o MEEM conforme o nível educacional dos idosos foi verificado que a média do MEEM para idosos sem escolaridade foi de $14,87 \pm 3,90$ pontos, para média escolaridade de $18,83 \pm 4,90$ pontos e alta escolaridade com média de $18,50 \pm 9,19$ pontos. (Tabela 2)

A alteração cognitiva foi avaliada conforme a escolaridade dos idosos, 25 idosos apresentou possível declínio cognitivo de acordo com o desempenho no MEEM, ou seja, 37,87\% dos idosos do presente estudo apresentam sintomas sugestivos de déficit cognitivo.

\section{Discussão}

As características identificadas no presente estudo são semelhantes às características encontradas em outros estudos com a mesma população alvo. A população idosa residente nas ILPS é predominantemente do gênero feminino em vários estudos. Segundo Groenwald et $\mathrm{al}^{16}$, Guedes et $\mathrm{al}^{17}$ e Micanto et $\mathrm{al}^{18}$, em pesquisas realizadas em instituições asilares de Canoas/RS, Passo Fundo/RS e Caxias do Sul/RS, respectivamente, houve prevalência de mulheres. Ainda, no mesmo estudo em Caxias do Sul, a média de idade $(78,3 \pm 9,3$ anos) é muito semelhante com o presente estudo. ${ }^{18} \mathrm{Na}$ literatura estrangeira o mesmo é observado, conforme estudos realizados em Madri e no Canadá (ref B e C)

Conforme estudo de Gualhardo et.al ${ }^{19}$, realizado em Pouso Alegre/MG, há prevalência de idosos caucasianos (74\%), e a maioria é católico (44\%), o mesmo predomínio foi encontrado nos idosos do presente estudo.

Num estudo com idosos institucionalizados na cidade de Presidente Prudente/SP, realizado por Converso et al, mostraram que $46,55 \%$ dos idosos são solteiros, $21,73 \%$ são viúvos, dados que coincidem parcialmente com os resultados deste estudo, em que o predomínio foi de idosos viúvos e solteiros. ${ }^{20}$

Num estudo realizado com idosos institucionalizados na cidade de Rio Claro/SP, por de Quadros et al verificou a escolaridade por gênero dos idosos. Nas mulheres, a média de anos em estudos foi de $5,36 \pm 2,61$ anos e nos homens 3,75 $\pm 1,84$ anos..$^{21}$ Analisando a escolaridade no presente estudo, as mulheres obtiveram média de anos em estudos de 2,67 $\pm 2,1$ anos e os homens $3,86 \pm 3,23$ anos (valor $p=0,076$ ), percebe-se diferença de maior escolaridade por gênero entre os estudos.

Referente ao uso de antidepressivos, em um estudo com idosos institucionalizados em Salvador/BA, realizado por Santana et al, 95,5\% dos idosos afirmaram que não faziam uso de medicação antidepressiva. ${ }^{22}$ No presente estudo, foi de $56,1 \%$ dos idosos que afirmaram que não faziam uso. Tal diferença, mesmo que seja a maioria dos idosos, pode ser explicada porque no presente estudo, além da resposta sim ou não, o idoso dispunha responder que não sabe quais medicações utiliza. Ainda, dependendo da forma como é perguntado, muitos idosos confundem medicações antidepressivas, com medicações ansiolíticas (benzodiazepínicos). Quanto ao hábito de fumar, em trabalho realizado por Del Duca et al na cidade de Pelotas/RS, foram identificados $63,1 \%$ de idosos não fumantes, $27,5 \%$ ex-fumantes e 9,4\% fumantes $9,4 .{ }^{23}$ Dados semelhantes encontrados no presente estudo, com idosos institucionalizados em Tubarão e Laguna, onde 56,1 \% são não-fumantes.

Quanto à prevalência de depressão, os resultados indicam alta taxa de sintomas depressivos nos ido-

Tabela 2

Análise do MEEM conforme escolaridade dos idosos

\begin{tabular}{lccc}
\hline Escolaridade & N & Média do MEEM & Desvio-padrão \\
\hline Sem escolaridade (0 anos de estudos) & 8 & 14,88 & 3,91 \\
Média escolaridade (1 a 8 anos de estudos) & 56 & 18,84 & 4,92 \\
Alta escolaridade (Mais que 8 anos de estudos) & 2 & 18,50 & 9,19 \\
\hline
\end{tabular}

Valor $\mathrm{p}=0,111$ 
sos da população estudada, $62,1 \%$ detectada pela EDG-15. Achados semelhantes foram encontrados em estudo na cidade de Pouso Alegre/MG e Curitiba/ PR. ${ }^{19,24}$

A prevalência de sintomas depressivos é maior em idosos institucionalizados quando comparados com idosos da comunidade. Num estudo realizado em Montes Claros/MG com idosos da comunidade, evidenciou apenas $20 \%$ de prevalência de sintomas depressivos. Com isso, verifica-se que a institucionalização pode constituir um fator de risco para depressão. ${ }^{25}$

Quanto à prevalência de sintomas depressivos por sexo, há maior prevalência de sintomas depressivos em indivíduos do sexo feminino, observando-se em alguns estudos, porém não foi observada associação estatisticamente significativa no presente estudo. $22,26,27,28$

Idosos que utilizam medicação antidepressiva obtiveram maior prevalência de sintomas depressivos, evidenciando que mesmo recebendo a medicação, a presença de sintomas depressivos ainda persiste. Logo, em tais idosos deveria ser realizada nova abordagem e caso seja necessário, ajustar a dose ou modificar a medicação.

A avaliação do déficit cognitivo nos idosos institucionalizados através do MEEM é amplamente utilizada. Comparando a pontuação do MEEM em outros trabalhos com idosos, verificaram-se dados semelhantes encontrados em Viçosa/MG, onde a média do MEEM foi de 19,48 pontos, porém aplicados aos idosos da comunidade ${ }^{29}$. No presente estudo, a média do MEEM foi de 18,34 $\pm 5,0$ pontos aplicados aos idosos institucionalizados.

Num estudo com idosas institucionalizadas em Curitiba/PR, verificou-se a media do MEEM de $16,68 \pm 5,60$ pontos. As mulheres no presente estudo apresentam média do MEEM de 17,72 $\pm 5,20$ pontos. $^{24}$

Quanto à prevalência de possível déficit cognitivo, no estudo com idosas institucionalizadas em Curitiba/ PR, utilizando os mesmo pontos de corte do presente estudo, verificou-se prevalência de $26,5 \%$, enquanto no presente estudo $37,87 \% .{ }^{24}$ Outros estudos, com idosos da comunidade encontraram menor prevalência de sintomas sugestivos de déficit cognitivo. ${ }^{25,30}$ Pode-se identificar que idosos institucionalizados apresentam maior alteração cognitiva e suspeita-se que a alteração cognitiva possa ser um dos motivos da institucionalização, ou então que a institucionalização é um fator de risco para possíveis declínios cognitivos, porém para afirmar tais circunstâncias novas pesquisam deferiam ser realizadas.

Como limitação do presente estudo pode citar a alta taxa de perdas. A exclusão de idosos por condições que possam interferir no entendimento é uma característica presente em estudos cuja população alvo envolve indivíduos de idade mais avançada. Esta condição por si só, pode ser considerada como uma subestimativa das prevalências aqui apresentadas, pois os idosos com alguma doença mental mais séria já instalada, não responderam o questionário.

\section{Conclusão}

O presente estudo evidenciou alta prevalência de sintomas depressivos nos idosos institucionalizados, onde $62,1 \%$ apresentaram sintomas depressivos. Ainda, foi verificada alta prevalência de possível déficit cognitivo nos idosos da pesquisa, representando $37,87 \%$ da amostra estudada.

Diante dos resultados da pesquisa e do número crescente da população idosa é essencial que os serviços de Saúde do Brasil estejam preparados para acolher, avaliar e tomar as medidas necessárias a fim de melhor avaliar saúde mental dos idosos brasileiros.

Desta forma, fazem-se necessárias a realização de intervenções do poder público a fim de apoiar as instituições de longa permanência para detectar precocemente as alterações cognitivas e a prevalência de sintomas depressivos no sentido de contribuir para uma melhor qualidade de vida desta população. Além disso, também é necessário que as próprias instituições de ensino capacitem melhor os profissionais que irão atuar juntamente a estas ILP.

\section{ABSTRACT}

Background: The process of aging is associated with the diseases that may compromise the proper functioning of the central nervous system. Objectives: To evaluate the prevalence of cognitive decline and depressive symptoms and their relationship with sociodemographic characteristics of institutionalized elderly in three long-stay institutions in south of Santa Catarina. Methods: cross-sectional study was carried out with all institutionalized elderly in Tubarão and Laguna (SC). In this study were interviewed 66 individuals, 60 years of age or older, male and female. The data collection was performed through a standardized questionnaire with sociodemographic characteristics, the Geriatric Depression Scale in the reduced form (GDS-15) and Mini-Mental State Examination. Results: The study population was pre- 
dominantly female, widowed, mean age $77.3 \pm 9.9$ years old. Depressive symptoms were present in $62.1 \%$ of institutionalized elderly. The cognitive decline was present in $37.87 \%$ of the institutionalized elderly. Conclusion: High prevalence of depressive symptoms and cognitive deficits were present in the elderly living in long-stay institutions surveyed. Given these facts, the health service in Brazil should be prepared to evaluate and treat institutionalized elderly, improving the quality of life of elderly patients.

Keywords: Elderly. Neuropsychiatric Disorders. Institutionalization. Depression. Cognitive deficit.

\section{Referênclas Blbllográficas}

1. Souza RF, Skubs T, Brêtas ACP. Envelhecimento e família: uma nova perspectiva para o cuidado de enfermagem. Rev Bras Enferm. 2000;60:263-7.

2. Alves GC, da Silva Júnior GB, Lima RSA, Sobral JB, Mota RMS, de Abreu KLS, Rocha NA et al. Fatores de risco para óbitos em pacientes idosos gravemente enfermos. Rev. Bras. Ter. intensiva 2010;22:138-43.

3. Maués CR, Paschoal SMP, Jaluul O, Omar CC, Jacob Filho W. Avaliação da qualidade de vida: comparação entre idosos jovens e muito idosos. Rev. Bras Clin Med São Paulo 2010;8:405-10.

4. Argimon II, Stein LM. Habilidades cognitivas em indivíduos muito idosos: um estudo longitudinal. Cad Saude Publica 2005; 21: 64-72.

5. Stella F, Gobbi S, Corazza DI, Costa JLR. Depressão no idoso: diagnóstico, tratamento e benefícios da atividade física. Motriz, Rio Claro 2002;8:91-8.

6. Reis, LA, Torres GV. Déficit cognitivo como fator de risco para a limitação de atividades cotidianas em idosos institucionalizados. C\&D-Revista Eletrônica da Fainor, Vitória da Conquista 2010;3:95-107.

7. Leite BFT, Salvador DHY, Araújo CL de O. Avaliação cognitiva dos idosos institucionalizados. Revista Kairós, São Paulo 2009;12:247-56.

8. World Health Organization (2012) disponível em: http:// www.who.int/mental_health/management/depression/ definition/en/index.html (consultado em 20 de fevereiro 2012)

9. Yesavage JA, Brink TL. Development and Validation of a Geriatric Depression Screnning Scale: A Preliminary Report. J Psychiatr Res. 1983;17:37-49.

10. Folstein MF, Folstein SE, McHugh PR. Mini-mental state: a practical method for grading the cognitive state of patients for the clinician. J Psychiatr Res1975; 12: 189-98.

11. Bertolucci PHF, Brucki SMD, Campacci SR, Juliano Y. O miniexame do estado mental em uma população geral: impacto da escolaridade. Arq Neuro-Psiquiatr. 1994; 52: 1-7.

12. Mincato PC, Freitas CLR. Qualidade de vida dos idosos residentes em instituições asilares da cidade de Caxias do SulRS. RBCEH 2007 jun; 127-138.

13. Papaléo Netto M. Gerontologia: a velhice e o envelhecimento em visão globalizada. São Paulo: Atheneu; 1999. 160-73.

14. Parmelle PA, Kleban MH, Lawton MP, Katz IR. Depression and cognitive change among institutionalized aged. Psychol Aging. 1991; 6:504-11

15. Almeida OP, Almeida AS. Confiabilidade da versão brasileira da escala de depressão em geriatria (GDS) versão reduzida. Arq Neuro Psiquiatr. 1999; 57(2B):421-6.

16. Groenwald RF. A institucionalização do idoso no município de Canoas-RS. Dissertação (Curso de Mestrado em Saúde Coletiva) - Universidade Luterana do Brasil, Canoas, 2003.

17. Guedes FM, Silveira RR. Análise da capacidade funcional da população geriátrica institucionalizada na cidade de Passo Fundo-RS. Revista Brasileira de Ciências do Envelhecimento Humano 2004;10:10-21.
18. Mincato PC, Freitas CLR. Qualidade de vida dos idosos residentes em instituições asilares da cidade de Caxias do Sul RS. RBCEH 2007;4:127-38.

19. Lacny C, Kirk A, Morgan DG, Karunanayake C. Predictors of cognitive impairment severity in rural patients at a memory clinic. Can J Neurol Sci 2012;39:774-81.

20. Pedrero-Chamizo R, Albers U, Tobaruela JL, Meléndez A, Castilho MJ, González-Gross M. Physical strengh is associated with Mini-mental state examination scores in Spanish institutionalized elderly. Geriatr Gerontol Int 2013; mar 19 (epud ahead of print).

21. Galhardo VAC; Mariosa MAS; Takata JPI. Depressão e perfis sociodemográfico e clínico de idosos institucionalizados sem déficit cognitivo Ver. Méd. Minas Gerais 2010; 20(1).

22. Converso MER, lartelli I. Caracterização e análise do estado mental e funcional de idosos institucionalizados em instituições públicas de longa permanência. J Bras Psiquiatr. 2007;56:267-72.

23. Quadro Junior AC, Santos RF, Lamonato ACC, Toledo NAS, Coelho FGdeM, Gobbi S. Estudo do nível de atividade física, independência funcional e estado cognitivo de idosos institucionalizados: análise por gênero. Brazilian Journal of Biomotricity 2008;2:39-50.

24. Santana AJ, Filho JCB. Prevalência de sintomas depressivos em idosos institucionalizados na cidade do Salvador. Revista Baiana de Saúde Pública 2007;31:134-46.

25. Del Duca GF, da Silva SG, Thumé E, Santos IS, Hallal PC. Indicadores da institucionalização de idosos: estudo de casos e controles. Rev Saúde Pública 2012;46:147-53.

26. Lenardt MH, Michel T, Borghi AdaS, Seima MD. O desempenho de idosas institucionalizadas no miniexame do estado mental. Acta Paul 2009;22:638-44.

27. Hoffmann EJ, Ribeiro F, Farnese JM, Lima EWB. Sintomas depressivos e fatores associados entre idosos residentes em uma comunidade no norte de Minas Gerais, Brasil. J Bras Psiquiatr. 2010;59:190-7.

28. Porcu M, Scantamburlo VM, Albrecht NR, Silva SP, Vallim FL, Araújo $\mathrm{CR}$ et al. Estudo comparativo sobre a prevalência de sintomas depressivos em idosos hospitalizados, institucionalizados e residentes na comunidade. Acta Scentiariun 2002;24:713-7.

29. Sass A, Gravena AAF, Pilger C, Mathias TAdeF, Marcon SS. Depressão em idosos inscritos no Programa de Controle de hipertensão arterial e diabetes mellitus. Acta Paul Enferm. 2012;25:80-5.

30. Carreira L, Botelho MR, de Matos PCB, Torres MM, Salciv MA. Prevalência de depressão em idosos institucionalizados. Rev. enferm. UERJ, Rio de Janeiro, 2011; 19:268-73

31. Machado, JC, Ribeiro R de CL, Leal PF da G, Cotta RMM. Avaliação do declínio cognitivo e sua relação com as características socioeconômicas dos idosos em Viçosa-MG. Rev. bras. epidemiol. 2007;10:592-605.

32. Mendes-Chiloff CL, Torres AR, Lima MC, Ramos-Cerqueira AT. Prevalence and correlates of cognitive impairment among the elderly in a general hospital. Dement Geriatr Cogn Disord 2009;28:442-8. 